\title{
A influência dos arranjos dentários em prótese total: revisão de literatura
}

The esthetic influence of the dental arrangements in complete denture: literature review

Lidia Audrey Rocha Valadas Marques Departamento de Odontologia Restauradora, Universidade Federal do Ceará, Fortaleza, Brasil.

Mara Assef Leitão Lotif Departamento de Odontologia Restauradora, Universidade Federal do Ceará, Fortaleza, Brasil.

Juliana Borges Gomes Departamento de Odontologia Restauradora, Universidade Federal do Ceará, Fortaleza, Brasil.

Rita Helena Mendes Garcia Lopes Departamento de Odontologia Restauradora, Universidade Federal do Ceará, Fortaleza, Brasil.

Mara Kelly Oliveira Silva Departamento de Odontologia Restauradora, Universidade Federal do Ceará, Fortaleza, Brasil.

José EugênIo TEIXEIRA Rocha Departamento de Odontologia Restauradora, Universidade Federal do Ceará, Fortaleza, Brasil.

\begin{abstract}
RESUMO
Introdução: A estética em prótese total aborda diversos fatores que proporcionarão a harmonia da boca e da face. O tamanho, forma e cor dos dentes são fatores importantes que devem ser considerados para uma correta seleção dos dentes artificiais. Além disso, a disposição dos dentes nos arcos é um fator imprescindível na elaboração de uma prótese mais estética, tornando-a mais natural. Objetivo: O objetivo deste artigo foi realizar uma revisão de literatura abordando os arranjos dentários utilizados em prótese total. Material e Métodos: Foram utilizados os bancos de dados Bireme, Medline e Scielo para pesquisa de artigos publicados entre os anos 1955 e 2013. Resultados: O arranjo e a montagem dos dentes em próteses totais é um assunto de muita controvérsia na literatura em virtude da existência de diferentes teorias para sua realização. Estudos que avaliam o arranjo dentário nas próteses totais, para obterem uma verdadeira avaliação da preferência da montagem pelo paciente, devem considerar que os dentes precisam ser arquitetados em conjunto com a face, uma vez que este conjunto (face e dentes) está intimamente ligado com a harmonia facial. Conclusão: A estética em prótese total tem sido um fator extremamente relevante para o sucesso e satisfação do paciente, existindo várias técnicas que possibilitam a determinação do arranjo. Essa escolha dependerá do bom senso do profissional, contribuindo com a necessidade e a vontade do paciente.
\end{abstract}

Palavras-chave: Prótese Total, Estética Dentária, Reabilitação Bucal. 


\begin{abstract}
Introduction: The aesthetic denture covers various factors that provide the harmony of mouth and face. The size, shape, and color of the real teeth are important factors that should be considered for a correct selection of the artificial teeth. Moreover, their arrangement in the arches is an essential factor in the development of an aesthetic prosthesis, making it more natural. Objectives: The purpose of this article was to review the literature regarding dental arrangements used in complete dentures. Methods: We searched Bireme, Medline, and SciELO databases for articles published between 1955 and 2013. Results: Teeth arrangement and assembly in complete dentures is a subject that raises much controversy in literature due to the existence of different theories for its making. In order to make a true assessment of the patient's preference, studies that assess tooth arrangement in complete dentures should consider that teeth must be designed having the whole face in mind, since face and teeth are closely connected with facial harmony. Conclusion: In complete denture, aesthetics has been an extremely important factor for success and for the patient's satisfaction, and there are several techniques as to how the design must be made. This choice will depend on the professional's good sense, contributing to the patient's needs and wishes.
\end{abstract}

Keywords: Complete Denture, Mouth Rehabilitation, Dental Aesthetics

\section{INTRODUÇÃO}

A restauração estética do desdentado tem um importante efeito psicológico, melhorando a sua autoestima e autoconfiança e, portanto, é uma parte fundamental do tratamento reabilitador oral. ${ }^{1}$

A estética em prótese total é um assunto que aborda diversos fatores que, em conjunto, proporcionam a harmonia da boca com o restante da face. Para a obtenção da estética favorável, é preciso considerar uma tomada correta da dimensão vertical, do plano oclusal protético, da linha mediana, da linha dos caninos e da linha alta do sorriso; com relação aos dentes, a forma, o tamanho e a cor são os fatores mais importantes a serem considerados para a correta seleção dos dentes artificiais. Além da forma, a disposição dos dentes é um fator imprescindível na elaboração de uma prótese mais estética, tornando-a mais natural. ${ }^{2}$

O conceito do belo é de caráter pessoal e subjetivo, sofrendo influências culturais e sociais. A demanda do paciente deve ser levada em consideração e sempre respeitada, permitindo um tratamento funcional com característica individual, que pode ser buscada por meio da utilização de diferentes arranjos dentários. Sendo assim, o objetivo do presente trabalho foi realizar uma revisão de literatura abordando os arranjos dentários utilizados em prótese total.

\section{Metodologia}

Para realização de uma revisão de literatura, foram utilizados os bancos de dados da Bireme, Medline e Scielo em busca de artigos publicados entre 1955 e 2013, em língua inglesa e portuguesa. Foram utilizados como descritores os termos: prótese total, estética dentária e reabilitação bucal. Foram obtidos 43 artigos e selecionados 21 para uso nesta revisão.

\section{REVISÃO DE LITERATURA}

A obtenção de uma estética adequada em prótese total está relacionada a um conjunto de fatores que resultam em harmonia facial para os pacientes. Porém, o desafio para confecção de tais próteses envolve a combinação da estética com a função e o conforto.

Para Vig, ${ }^{3}$ a estética está tão relacionada psicologicamente com o conforto que ambos não podem estar separados. Isto é, a confiança que o paciente tem na capacidade da prótese permanecer em posição e não ser identificada por outras pessoas está intimamente ligada à aparência. 
Em estudo realizado por Brewer, ${ }^{4} 12$ pacientes receberam sete próteses totais, as quais se diferenciavam pelo arranjo dentário e tipo de material utilizado na confecção dos dentes. Além disso, foram confeccionadas por profissionais diferentes. Os resultados mostraram que 11 dos participantes escolheram a prótese com melhor aparência em detrimento do conforto e função.

Um fator importante para construir uma prótese estética, além da forma, cor e tamanho dos dentes artificiais, é o arranjo destes, ou seja, a disposição na prótese. Outros fatores importantes são: consulta inicial para conhecimento da expectativa do paciente, tomada precisa da oclusão cêntrica, disposição do plano oclusal e a influência da própria instalação e manutenção. ${ }^{2}$

Um estudo realizado por Pereira ${ }^{5}$ com 49 universitários dentados, sendo 20 do gênero masculino e 29 do gênero feminino, com média de idade de 21 anos, comparou as técnicas existentes na literatura para identificar qual método proporciona a seleção de dentes artificiais com dimensões mais próximas dos dentes naturais e concluiu que o arranjo adequado dos dentes aumenta a estabilidade da prótese, o conforto, a estética e a função.

Os estudos de Waliszewski ${ }^{6}$ apresentam teorias para a escolha do arranjo dos dentes artificiais. A teoria temperamental, ou da correspondência e harmonia, introduzida em 1872, e que relacionou cor e forma dos dentes em harmonia com a idade e o gênero do paciente, baseou-se na proposição de que a personalidade do sujeito e as características corporais combinam com o tamanho, proporção, disposição e cor dos dentes. A teoria geométrica, desenvolvida em 1914, relaciona a forma da face com a forma dos dentes, definindo três formatos dentários básicos: ovoide, quadrado e triangular. Segundo o autor, outras pesquisas demonstraram que não existe correlação significativa entre a forma facial e a forma dos dentes, pois há dificuldades relativas à idade, estilo de cabelo e alterações de massa corporal.

Em 1955, Frush e Fischer ${ }^{7}$ apresentaram $^{2}$ a teoria dentogênica. Médias populacionais para os sexos e idade foram a base para a seleção dos dentes, suas formas, tamanhos e proporções, arranjo e cor. Os estudos destes autores basearam-se em populações de pacientes edêntulos e não portadores de próteses. Tal teoria aborda os fatores gênero, personalidade e idade. Quanto ao gênero, diferencia-se o contorno dos ângulos incisais em retos e arredondados, para o sexo masculino e o feminino, respectivamente. Quanto à idade, em jovens há um desnível nas bordas incisais dos dentes anteriores superiores; já em idosos, deve haver uma simulação de desgastes fisiológicos, deixando as bordas incisais niveladas. Quanto à personalidade, classifica-se o tipo vigoroso para homens, médio para a maioria da população e delicado para mulheres. Porém, a percepção de uma personalidade é subjetiva, e estudos demonstram que diferentes arranjos dentários podem projetar diferentes traços de personalidade. . $^{2,6} 7$

Portanto, torna-se necessário considerar a opinião do paciente na escolha do melhor arranjo, pois a prótese deve refletir a personalidade deste, e não a do cirurgiãodentista ou do protético, devendo estes ser treinados para perceber as peculiaridades de cada indivíduo para não manter um padrão de arranjo dentário. ${ }^{8}$

O estudo de Marunick e colaboradores ${ }^{9}$ teve como objetivo observar a preferência do paciente quanto à forma dos dentes de sua prótese. Utilizaram a exibição de quatro fotografias, obtidas de três homens e de três mulheres desdentadas com diferentes apresentações da boca. Cada participante apresentava uma das formas clássicas da face: quadrada, triangular e oval. Foram 
selecionados $(n=110)$ voluntários inscritos na Faculdade de Odontologia, onde foi realizado o estudo. Foi-lhes perguntado qual a aparência mais agradável, sem que soubessem qual o arranjo dos dentes em cada foto. Uma das fotos exibia a face com a mandíbula em posição de repouso e a outra com um sorriso descontraído e aberto mostrando o arranjo dos dentes. O resultado obtido revelou que houve preferência por dentes quadrados, seguido dos dentes ovoides e, por último, os de formato triangular. A forma ovoide foi a mais aceita pelo sexo feminino, enquanto que o sexo masculino preferiu a forma quadrada.

Dong e colaboradores $^{10}$ avaliaram em pesquisa coreanos com oclusão normal por meio de fotografias obtidas com o sorriso aberto e sua qualidade estética de acordo com alguns quesitos, dentre eles a posição do lábio no sorriso. Os autores concluíram que os sorrisos mais atraentes tinham os dentes que seguiam a linha do lábio inferior, exibiam todo o comprimento dos incisivos centrais superiores sem exposição gengival e eram simétricos.

Castro Jr. et al. ${ }^{2}$ avaliaram cinco tipos de montagens de dentes em dez voluntários desdentados totais do sexo masculino, com média de idade de 59,1 anos. Para cada montagem foi modificada a posição dos dentes e alguns desgastes nos dentes anteriores superiores foram realizados para personalizar as próteses que ocluíam com uma prótese total inferior padrão. As montagens dos arranjos foram: (1) montagem clássica ou jovem com desníveis entre as bordas dos incisivos centrais e laterais, correspondendo aos arcos dentais de indivíduos na primeira fase da maturidade; (2) montagem com toque masculino na qual os incisivos centrais superiores eram expostos, projetando-se o lado distal para vestibular e os incisivos laterais superiores eram deslocados suavemente para a face palatina, tornando-os menos evidentes. Esses arranjos evidenciam os incisivos centrais superiores e os caninos, tornando o aspecto facial circunspecto; (3) montagem com toque feminino, na qual a distal dos incisivos centrais superiores são ligeiramente deslocadas para a palatina e os ângulos mésio-incisais dos incisivos laterais superiores são sobrepostos sobre a face vestibular dos incisivos centrais superiores, colocando-os em evidência; (4) montagem com diastemas em que as faces proximais dos incisivos centrais e incisivos laterais superiores foram desgastadas; (5) montagem senil com as bordas incisais dos incisivos centrais, incisivos laterais e caninos superiores desgastadas, deixando-os com ângulos mais vivos e nivelados, simulando desgastes fisiológicos. Os resultados de satisfação mostraram que a montagem clássica e senil foram as que mais agradaram, com $38,57 \%$ e $32,85 \%$, respectivamente, sem apresentar diferenças estatísticas significantes entre eles. E, com menor agrado, foram a com toque feminino, com $32,86 \%$ de desaprovação, seguida da montagem com toque masculino $(31,43 \%)$ e com diastema $(22,86 \%)$, embora não tenham ocorrido diferenças estatísticas significantes entre as montagens com toque feminino, com toque masculino e com diastemas.

Em um estudo realizado por Waliszewski e colaboradores, ${ }^{6}$ fotografias frontais coloridas dos participantes sorrindo, com os diferentes arranjos, foram agrupadas em um catálogo para ser avaliado por 147 desdentados totais, dos quais 88 eram do gênero feminino e 59, do gênero masculino. O resultado obtido foi que $55 \%$ preferiu a aparência natural, $26 \%$, a aparência protética e $19 \%$ dos avaliadores preferiram a supernormal. Quando foram analisados os quesitos idade, gênero, objetivo do paciente (estética ou função), as porcentagens não tiveram mudanças estatisticamente significativas.

Em 2006, Waliszewski e colaboradores ${ }^{11}$ realizaram uma pesquisa de preferência estética com seis pacientes edêntulos com 
idade média de 61,2 anos. Três arranjos de montagem de dentes para prótese total foram confeccionados para cada um. Dezoito fotografias com sorriso aberto foram tomadas de cada voluntário. Foi escolhido um participante do gênero masculino e um do gênero feminino para cada faixa etária: inferior a 30 anos, meiaidade (50 anos) e superior a 70 anos. Cada arranjo representou um conceito estético: aparência natural, aparência supernormal e aparência protética. O conceito natural foi definido como um aspecto determinado pelo caráter e inerente ao biótipo do indivíduo. Em contrapartida, o supernormal afasta-se do que é habitual ou normal, pois transcende as leis da natureza. Por último, o aspecto protético, que representa a caracterização típica de usuários de prótese total.

Stockheimer e colaboradores ${ }^{12}$ realizaram pesquisa semelhante, porém entrevistando a preferência de 269 desdentados e de 167 dentados diante dos mesmos três conceitos estéticos. Utilizaram o mesmo catálogo com a combinação de fotos e arranjo do trabalho anterior. Os resultados obtidos demonstraram não existir diferença significativa na preferência estética para ambos os grupos.

Em estudo recente, Katase e

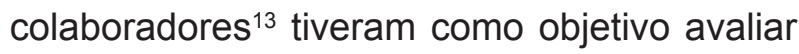
a precisão de um método para a simulação do rosto de um portador de dentaduras completas. A metodologia simulou integralmente 0 aspecto facial de dez desdentados, dentre os quais três eram do gênero masculino e sete do feminino. Em seguida, foi trocado o arranjo da montagem dos dentes, resultando em cinco próteses totais elaboradas em um computador, com auxílio do software CAD/ CAM. De acordo com a metodologia utilizada no estudo, os dados sugeriram que não foram encontradas diferenças significativas entre as características faciais dos aspectos simulados e as faces reais com dentaduras prototipadas. Assim, o método de simulação computadorizada das características do aspecto facial para a confecção de próteses totais pode ser clinicamente útil.

\section{Discussão}

Confeccionar próteses totais que restabeleçam a função, a estética e que permitam conforto durante o uso é um grande desafio para o profissional. A estética é avaliada de maneira subjetiva, de acordo com cada indivíduo, e a função e o conforto dependem do correto planejamento das próteses. Para ser considerado esteticamente agradável, o arranjo dos dentes artificiais deve ser tal que promova harmonia facial ao paciente, observando variáveis como a cor da pele, do cabelo e dos olhos, os traços de expressão facial e demais detalhes anatômicos individuais. Contudo, o arranjo na montagem dos dentes em próteses totais ainda é um assunto controverso na literatura em virtude da existência de diferentes teorias para sua realização.

A teoria temperamental foi provavelmente a primeira a descrever o aspecto estético em odontologia. ${ }^{14}$ Esta teoria observa variáveis como a cor e a forma dos dentes em harmonia com a idade e o gênero, baseada na ideia de que a personalidade do sujeito e as características corporais combinavam com o tamanho, proporção, disposição e cor dos dentes. A impossibilidade de se chegar a um acordo sobre a caracterização de uma prótese total para cada personalidade, ou de se decidir quais personalidades existem, limitou a utilidade prática desta teoria.

Estudos de Bell' ${ }^{15}$ e Mavroskoufis e Ritchie $^{16}$ sugeriram que não há relação entre a forma da face e a forma do incisivo central superior em pacientes dentados, contrariando a teoria de Williams.

Cabe ressaltar que a raça também influencia na forma do incisivo central superior. Estudos de Cesário Jr. e colaboradores ${ }^{17}$ observaram 
uma relação entre as diferenças raciais e sexuais, nas quais as mulheres e os homens negros apresentaram medidas maiores que os brancos, e as medidas nos homens eram, geralmente, maiores que nas mulheres, em ambas as raças.

A teoria dentogênica, introduzida por Frush e Fischer ${ }^{9}$ leva em consideração os fatores sexo, personalidade e idade para o arranjo dos dentes. Segundo Almeida e colaboradores, ${ }^{18}$ a teoria dentogênica é uma das mais difundidas, enfatizando que a estética está relacionada a estes três fatores.

Estudos que avaliam o arranjo da montagem dos dentes em prótese total para obterem a satisfação estética do paciente devem considerar que a forma e a aparência dos dentes precisam estar em harmonia com a face. Pesquisas que mostram apenas os arranjos das montagens dos dentes são limitadas, embora os arranjos perfeitamente alinhados são os de maior aceitação.

\section{Conclusão}

Diante do exposto, pode-se observar que a estética em prótese total tem sido um fator extremamente relevante para o sucesso e satisfação do paciente, mostrando-se em alguns estudos mais importante que a estabilidade e restabelecimento da função.

Observou-se que os arranjos dentários são fundamentais para o cumprimento dos pré-requisitos necessários para a satisfação, sendo de caráter pessoal, modificando-se de acordo com a vontade e a expectativa de cada indivíduo.

Existem várias técnicas, seguindo linhas diversas, como teoria dentogênica, teoria temperamental, montagens e forma dos dentes de acordo com a faixa etária de cada indivíduo, dentre outras, que possibilitam a determinação do arranjo que deverá ser realizado. Contudo, não há uma padronização universal dessas opções. Essa escolha dependerá do bom senso do profissional, contribuindo com a necessidade e a vontade do paciente.

A utilização de imagens do paciente e simuladores de rostos reais para a fabricação de próteses totais pode ser uma ferramenta de grande ajuda na escolha do arranjo ideal para cada paciente, entretanto, mais estudos são necessários para avaliar sua praticidade e a relação custo-benefício.

Estudos com amostras adequadas, randomizados e duplo-cegos são necessários para que se possa avaliar satisfatoriamente qual o arranjo mais harmonioso para cada paciente.

\section{REFERÊNCIAS BIBLIOGRÁFICAS}

1. Barbosa DB, Barão VAR, Assunção WG, Gennari Filho $\mathrm{H}$, Goiato MC. Complete denture insertion: A review. Rev Odontol Unesp, 2006; 35(1):53-60.

2. Castro Jr OV, Hvanov ZV, Frigerio MLMA. Avaliação estética da montagem dos seis dentes superiores anteriores em prótese total. Pesq Odont Bras, 2000; 14(2):177-82.

3. Vig RG. The denture look. J Prosthet Dent, 1961; 11(1):9-15.

4. Brewer A. Selection of denture teeth for esthetics and function. J Prosthet Dent,1970; 23(2):368-73.

5. Pereira MC, Lucas BL, Junior RB, Gonçalves LC, Gomes VL. Seleção de dentes artificiais: estudo comparativo da largura e do comprimento dos seis dentes anteriores superiores naturais com dentes artificiais. Fapemig, 2008; $1(1): 9-19$.

6. Waliszewski MP. Restoring dentate appearance: A literature review for modern complete denture esthetics. Journal of Esthetic and Restorative Dentistry, 2005; 93(1):386-94.

7. Frush JP, Fischer RD. Introduction to dentogenic restorations. J Pros Den,, 1955; 5 (1): 586-95.

8. Telles D. Prótese total convencional. São Paulo: Santos, 2009; pp. 70-8.

9. Marunick MT, Chamberlain BB, Robinsos CA. Denture aesthetics: An evaluation of laymen's preferences. J Oral Rehabil, 1983; 10(1):399-406. 
10. Dong JK, Jin TH, Cho HW, Oh SC. The esthetics of the smile: A review of some recent studies. Int J Prosthodont, 1999; 12(1):9-19.

11. Waliszewski MP, Shor A, Brudvik J, Raigrodski AJ. A Survey of edentulous patient preference among different denture esthetic concepts. Journal of Esthetic and Restorative Dentistry, 2006; 18(1):352-69.

12. Stockheimer C, Waliszewski MP. A survey of dentulous and edentulous patient preference among different denture esthetic concepts. Journal of Esthetic and Restorative Dentistry, 2012; 24(1):112-25.

13. Katase H, Kanazawa M, Inokoshi M, Minakuchi $\mathrm{S}$. Face simulation system for complete dentures by applying rapid prototyping. The Journal of Prosthetic Dentistry, 2013; 109(6):353-60.

14. Miraglia SS, Freitas KB, Pinto JHN. Análise comparativa das distâncias mésio-distal e gêngivo-incisal dos incisivos centrais superiores com a régua trubytetoothindicator. Rev Odontol, 2002; 5(2):13-7.
15. Bell RA. The geometric theory of selection of artificial teeth: is it valid? J Am Dent Assoc, 1978; 97(1):637-40.

16. Mavroskoufis F, Ritchie GM. The face-form as a guide for the selection of maxillary central incisors. J Prosthet Dent, 1980; 43(1):501-5.

17. Cesario Jr VA, Latta Jr. Relationship between the mesiodistal width of the maxillary central incisor and interpupillary distance. J Prosthet Dent, 1984; 52(1):641-3.

18. Almeida RKM, Magalhães MPM, Kaieda AK, KairallaSA, Torres FC, Paranhos LR. A forma do incisivo central superior na visão da literatura. Rev Fac Odontol, 2011; 16(3):337-41.

Autor correspondente:

Lidia Audrey Rocha Valadas Marques

Departamento de Odontologia Restauradora, Universidade Federal do Ceará, Fortaleza, Brasil. e-mail: lidiavaladas@gmail.com

Submetido em: 18-11-2013

Aceito em: 28-5-2014 